\title{
Motion Controlled Gait Enhancing Mobile Shoe for Rehabilitation
}

\author{
Ismet Handzic*, Erin V. Vasudevan ${ }^{\dagger}$, and Kyle B. Reed* \\ *Department of Mechanical Engineering, University of South Florida, Tampa, Florida, 33612 \\ ${ }^{\dagger}$ Moss Rehabilitation Research Institute, Elkins Park, PA, 19027
}

\begin{abstract}
Walking on a split-belt treadmill, which has two belts that can be run at different speeds, has been shown to improve walking patterns post-stroke. However, these improvements are only temporarily retained once individuals transition to walking over ground. We hypothesize that longer-lasting effects would be observed if the training occurred during natural walking over ground, as opposed to on a treadmill. In order to study such long-term effects, we have developed a mobile and portable device which can simulate the same gait altering movements experienced on a split-belt treadmill. The new motion controlled gait enhancing mobile shoe improves upon the previous version's drawbacks. This version of the GEMS has motion that is continuous, smooth, and regulated with on-board electronics. A vital component of this new design is the Archimedean spiral wheel shape that redirects the wearer's downward force into a horizontal backward motion. The design is passive and does not utilize any motors. Its motion is regulated only by a small magnetic particle brake. Further experimentation is needed to evaluate the long-term after-effects.
\end{abstract}

\section{INTRODUCTION}

Persons suffering central nervous system damage, such as stroke or individuals that have suffered damage to the spinal cord, brainstem, cerebellum, and motor cortex, sometimes develop an asymmetric walking pattern where one leg does not fully swing backward. This uneven gait hinders these individuals from properly and efficiently moving through everyday life. In persons with an asymmetric walking pattern, one leg does not move back far enough to achieve a correct gait.

A split-belt treadmill has been shown to alter the gait patterns [1][2][3][4][5] in individuals and various animals [6][7] with symmetric and asymmetric walking patterns. A split-belt treadmill has two belts traveling at different rates. This setup allows one foot to be repeatedly pushed backward faster than the other foot, which, after returning to the same speed treads, generates after-effects with a symmetric gait. Continuous and repeated correction through the use of a split-belt treadmill setup results in temporary restoration of a normal gait, but the walking pattern diminishes over time and only partially transfers to walking over ground [8]. Once individuals' gait was altered to a symmetric gait and transitioned to solid ground, there are only small signs of the corrected walking pattern that last for a brief period. Another aspect for these studies is that individuals only adapted over a short amount of time, hence effects from long-term training are still unknown.

An explanation of this transference problem involves context awareness [9], or location awareness. Context awareness is the human's ability to automatically account for perturbations to the physical body while preparing and adjusting for such disturbances. A good example of context awareness is the body's unconscious leaning forward when stepping onto a moving object such as an escalator [10][11]. It is hypothesized that this same type of context awareness is causing the corrected walking pattern to vanish once individuals set out to walk over ground. The individual's body readjusts and rebalances to different outside perturbations. A portable shoe, worn over ground, which can accomplish the same corrective movements, would likely overcome this context awareness problem and allow rehabilitation in the natural environment where the walking will occur. Such a shoe would also reveal long-term effects due to the same type of gait training.

This concept has evolved into the Gait Enhancing Mobile Shoe (GEMS), shown in Figure 1. The portable GEMS imitates the same relative foot motion experienced in previous split-belt treadmill gait rehabilitation methods, but while walking over ground.

The GEMS presented in this paper is the successor of a previous version [12]. The previous version was passive and had no control of the backward foot motion. The predecessor also moved the wearer's foot backward, but in a jerky and fairly unpredictable motion comparable to sliding on ice or a slippery surface. The GEMS version outlined in this paper is passive as well and utilizes the wearer's downward force by converting it into a horizontal backward motion. The force is redirected by using a specially sized Archimedean spiral wheel shape that actually pushes the wearer's foot backward. An on-board magnetic particle brake applies a resistance torque through an internal gear train which in turn controls the velocity of the shoe's backward motion. The shoe is designed to be powered by a small battery pack worn around the waist.

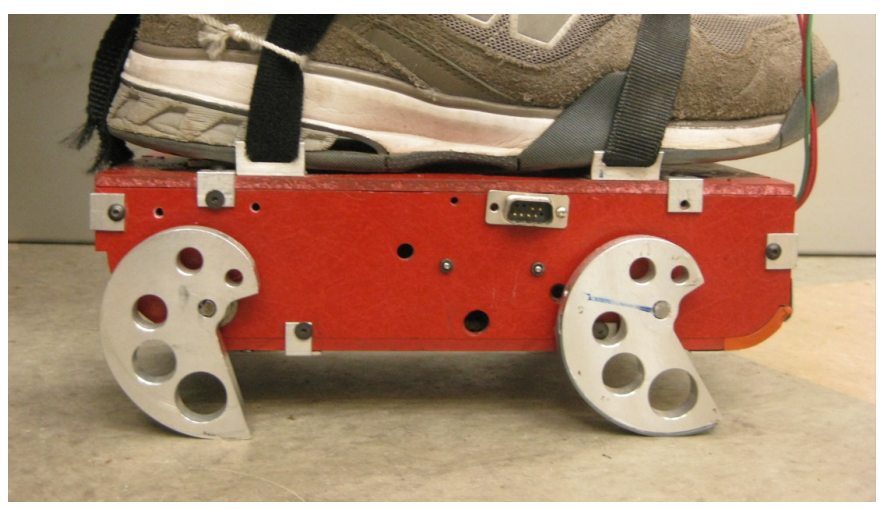

Fig. 1. Gait Enhancing Mobile Shoe (GEMS) 

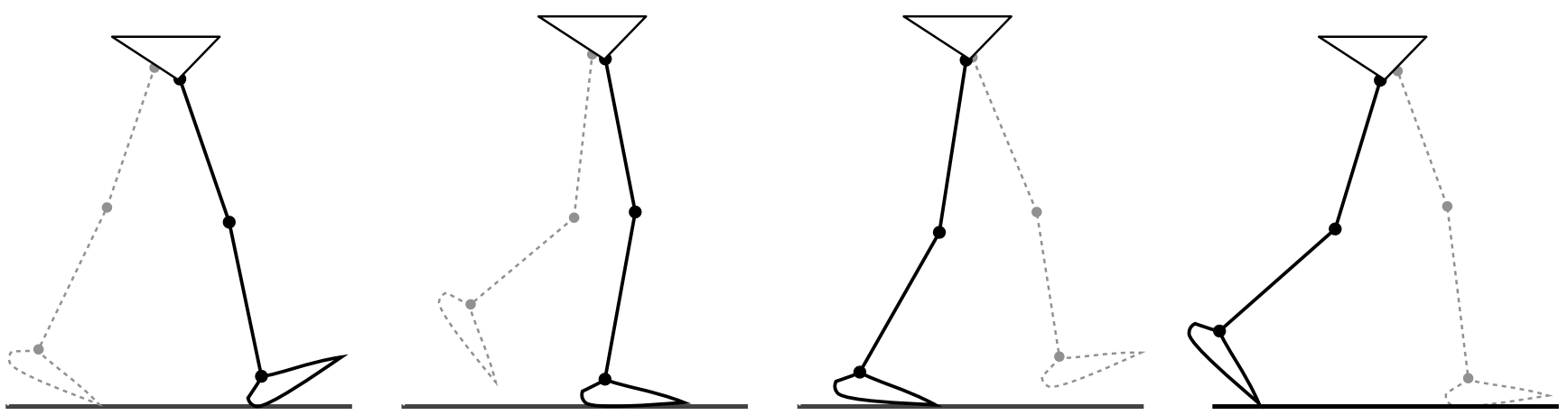

Fig. 2. Adult Human Walking Gait Cycle. Dark lines describe the stance phase while dotted lines represent the swing phase.

Advantages of such a portable rehabilitation device are that it can be worn in different environments including one's own home and also that it can be worn for an extended period of time, thus the corrected gait is predicted to persist longer than those of a split-belt treadmill. Moreover, the ability to wear the GEMS for longer periods of time increases the probability of producing better gait rehabilitation effects.

\section{BACKGROUND}

\section{A. Gait Phases and Reaction Forces}

It is important to point out the kinematics and other details of normal human gait before discussing the applications of the GEMS and its rehabilitation methods. Walking is an innate human ability to propel one's body forward or backward. The forward and backward walking motions are exactly similar in kinematics while only differing in EMG activity in various leg and foot muscles [13].

The kinematics of the human gait cycle can be divided into two distinct phases: the stance phase and the swing phase [14]. Furthermore, these two gait cycle phases can be broken down into seven gait phases, some of which are shown in Figure 2. The GEMS transfers the vertical force applied from the wearer's weight during the stance phase into a horizontal motion. Figure 3 shows the variable horizontal and vertical forces during the stance phase in an adult walking gait starting at heel contact. Notice that during the stance phase, a slightly fluctuating $800 \mathrm{~N}$ vertical force is applied, while horizontal reaction forces of up to $\pm 200 \mathrm{~N}$ are experienced. Also notice that at the $33 \%$ gait cycle mark, the horizontal reaction force switches from pushing the leg backward to pushing the leg forward.

The GEMS limits the total amount of motion relative to the ground so the balancing reflexes are not activated after heel contact. The GEMS also takes all instances leading up to the toe off and converts it to a controlled horizontal backward motion. It is stressed that the GEMS applies a controlled backward motion, which is desired so that balancing reflexes are not activated and a more natural walking transition is experienced.

\section{B. Correcting Gait}

Individuals that have suffered a stroke or damage to the central nervous system have been found to successfully adapt their walking pattern after only fifteen minutes on a split-belt treadmill [1][2][3][4][5]. However, these effects were shortlived and wear off quickly during over ground walking. Taking into account that these studies have been conducted over short periods of time, it is suggested that the long-term aspects of these after-effects should be studied. These long-term splitbelt treadmill effects are of course impossible to study with a stationary split-belt treadmill. Hence, a mobile device such as the GEMS can be utilized to observe such long-term effects by letting test subjects wear the GEMS shoe over an extensive period of time, monitoring the magnitude of the after-effects periodically.

Long-term effects could be trained by letting the test subject wear the shoe on the weak leg and so limiting the forward motion of the weak leg. This motivates the individual to lengthen the forward distance to initial heel contact. The

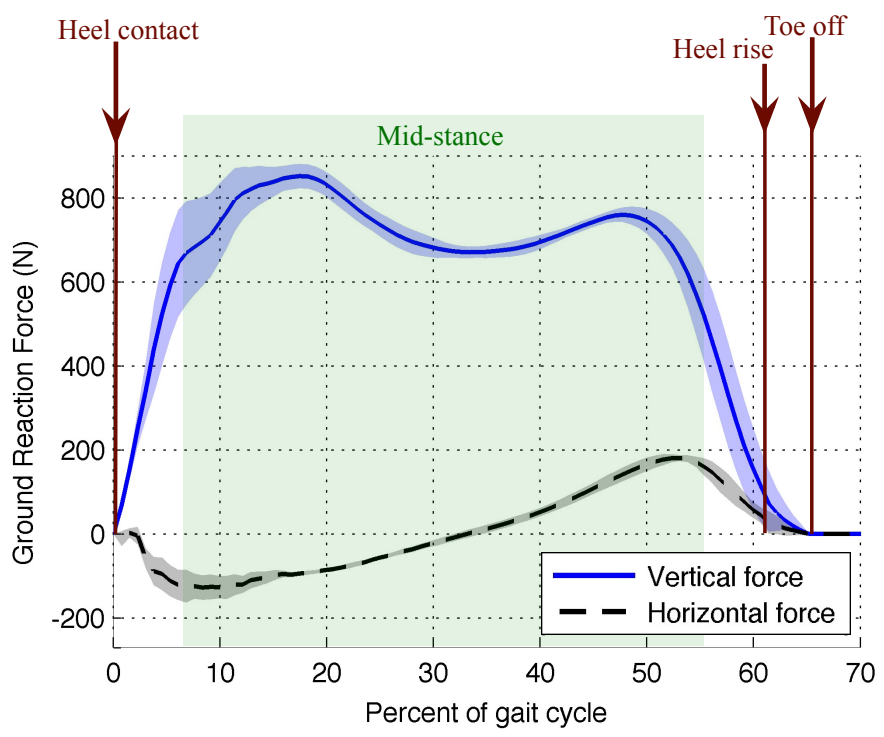

Fig. 3. The horizontal and vertical reaction forces change throughout the gait cycle. The GEMS uses these changing forces to alter gait patterns for rehabilitation. 
GEMS also pushes the leg backward in a controlled velocity simulating a correct gait. While the previous GEMS version abruptly pushed the foot back in an uncontrolled fashion, this revised GEMS design regulates the backward motion for a smooth transition between gait phases.

Although easily confused, the kinematics of walking on a split-belt treadmill with asymmetric belt velocities and walking with the GEMS differ slightly. On a split-belt treadmill, the body's relative velocity to the ground is zero, while each foot has a backward velocity. Wearing the GEMS the body's relative velocity is non-zero and forward, the foot without the GEMS has a zero velocity, and the foot wearing the GEMS has a backward velocity. However, velocities between both feet relative to the body are the same for both methods. We are using passive dynamic walkers to further evaluate the differences between walking on the GEMS, walking on a splitbelt treadmill, and walking over ground [15].

We hypothesize that prolonged wearing of the GEMS will have positive after-effects that help asymmetric walking patients adapt a more normal walking gait over a longer period of wearing the GEMS. This application of the GEMS also has the potential to increase muscle impedance through external perturbations [16], resulting in a beneficially altered gait.

Even if no permanent after-effects are found to be present, the GEMS can still be used to correct asymmetric walking patterns by wearing the shoe on the strong leg and letting it swing past the stance phase, limiting forward progression, and letting individuals toe off with that leg. As a result, both legs will push the individual forward by similar distances, evening out the asymmetric gait. This is important considering that post-stroke patients do not bring one leg back far enough, causing a limp and an unsuccessful toe off.

\section{GEMS and Context Awareness}

Context awareness is the human's ability to automatically account for perturbations to the physical body while preparing and adjusting for such disturbances [17]. The act of stepping onto a stationary escalator was analyzed and it was demonstrated that the body relies heavily on visual sensory and visual cues for balancing [18]. For example, it is much harder to balance one's own body on one foot with eyes closed compared to having one's eyes open. This suggests that the body's visual exteroception plays a dominant role in the gait training and in turn the adaptation of symmetric gait in hemiplegic patients. It is this context awareness that is hypothesized to be an integrating factor in the inability to store the previously described feed forward motion learned in splitbelt gait manipulation research [1]. While after-effects can be achieved [1][12], as subjects adapt to the asymmetric treadmill speed and subsequently walk over ground, the learned gait motion disappears quickly. The visual cues are distinctly different between walking on a treadmill and walking over ground.

The concept of the GEMS eliminates the problem of context awareness during the gait adaptation process. With the GEMS, the human gait is altered in the natural context of walking. The walking gait is slowly altered using the split-belt treadmill concept, however, during training there is no disconnect between the visual cues during the adaptation process and the visual cues after the adaptation process.

\section{GEMS DESIGN}

\section{A. Previous GEMS Design}

An existing Gait Enhancing Mobile Shoe (GEMS) prototype [12] has been developed that successfully generated the desired motion. Although the previous design was able to move the wearer's foot backward, it performed the motion in a jerky, uncontrolled, and unnatural manner. Also, instead of pushing the wearer's foot backward, it acted as if the wearer was slipping on ice or a slippery surface. It is assumed that this uncontrolled motion activates the body's balancing and recovery reflexes, thus hindering a positive adaptation of an altered walking pattern. In previous split-belt studies this was not an issue due to a consistent predictable asymmetric foot motion. In addition, as a result of the previous model's large horizontal backward motion of $10^{\prime \prime}(25 \mathrm{~cm})$, the walking speed was decreased. A huge limitation of the previous GEMS model was that it had little adjustability in backward motion velocity, travel distance, or travel direction.

\section{B. Motion Controlled GEMS Design}

While the broad concept of this version of the GEMS stems from the previous version, the new version is a complete redesign utilizing different mechanical concepts. The improved GEMS design aims to smoothen the transitions between phases in a human gait by having the possibility to regulate the horizontal backward motion of the foot. This controlled motion makes the redesigned GEMS similar to the foot motion experienced by the split-belt treadmill. The new redesign (shown in Figure 4) still acts in a passive manner in that it utilizes the wearer's vertical downward motion to create a horizontal backward motion.

1) Wheel Shape: A vital part of the GEMS design is its wheel shape. The wheels are designed based on the Archimedean spiral shape shown in Figure 5. The radius changes throughout the rotation angle, which is similar to rolling down a hill with a uniform wheel, but in this case the slope is attached to the foot and is not part of the ground. By using this Archimedean spiral shape, the wearer's applied vertical force $\left(F_{V}\right)$ during the stance phase is redirected to the instantaneous horizontal backward force $\left(F_{H}\right)$ through Equation 1,

$$
F_{H}=F_{V}\left(\frac{L}{R}\right),
$$

where $L$ is the instantaneous perpendicular distance between the wheel center and the ground contact point. The radius, $R$, over a whole rotation of an Archimedean spiral shape is obtained by Equation 2,

$$
R(\theta)=b \theta^{\frac{1}{n}},
$$

where the constants $b$ and $n$ constitute the size and shape of the Archimedean spiral. 


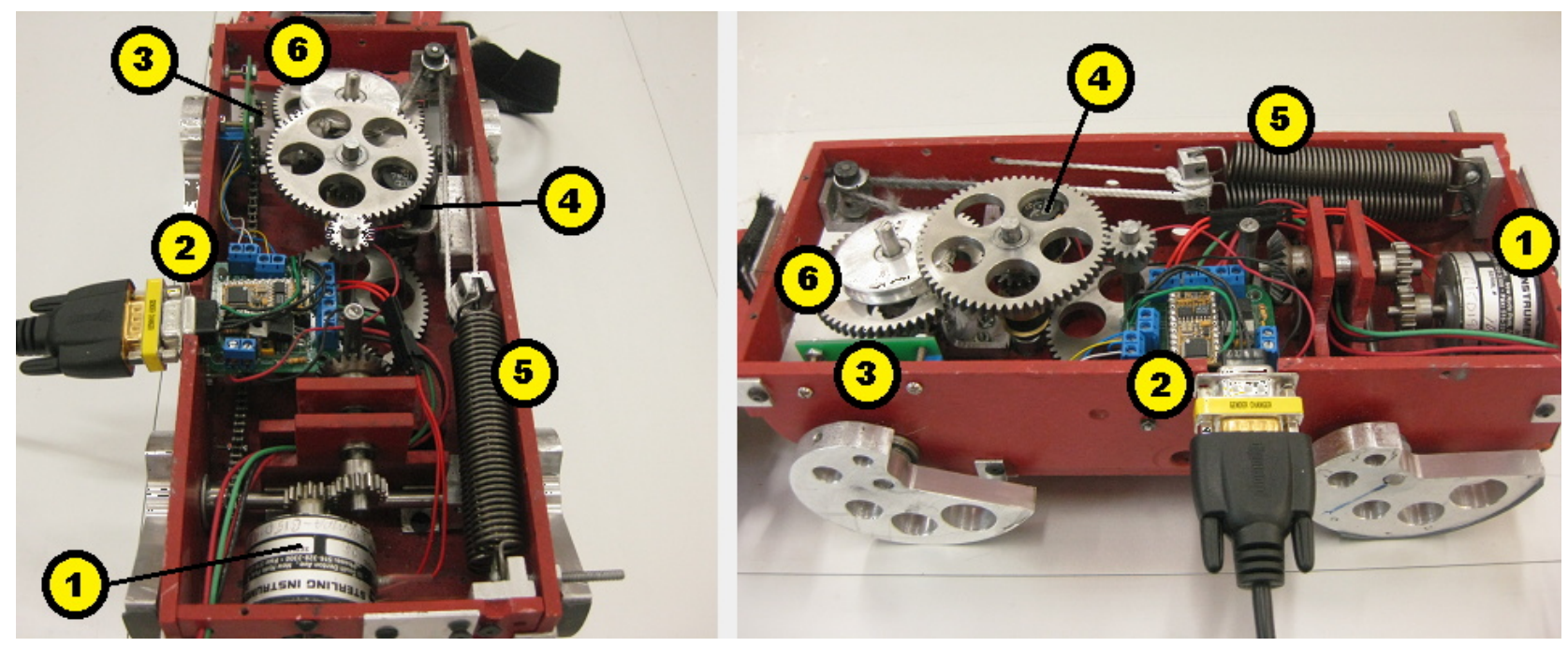

Fig. 4. GEMS interior: (1) Magnetic Particle Brake, (2) Microprocessor with RS232 Connection, (3) Accelerometer, (4) Potentiometer, (5) Reset Spring, (6) Reset Spring Pulley.

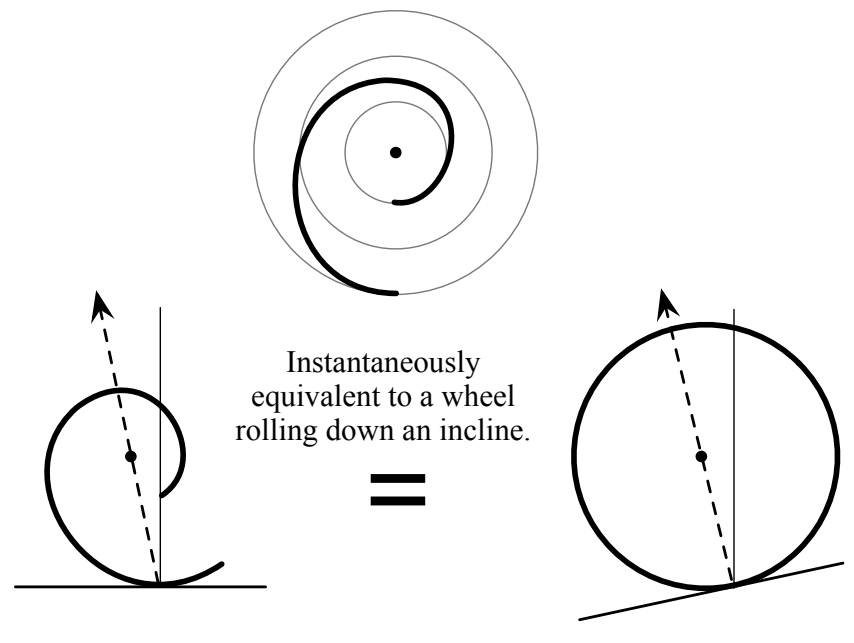

Fig. 5. The GEMS wheel shape is designed so that when normal vertical force is applied it behaves similar to rolling down a slope.

Using this wheel's shape allows the manipulation of several customized variables such as the horizontal travel distance, horizontal maximum velocity, overall shoe height, and the horizontal backward force exerted by the shoe onto the wearer's foot. Considering these variables, a wheel size with a largest radius of $2^{\prime \prime}$, shortest radius of $0.75^{\prime \prime}$, and $n$ constant of 1 were chosen to provide an average horizontal backward force of $160 \mathrm{~N}(36 \mathrm{lb})$ given an $800 \mathrm{~N}(180 \mathrm{lb})$ vertical downward force. The average horizontal force exerted by the wheel was found using Equation 3,

$$
F_{H, a v e}=\frac{1}{R_{2}-R_{1}} \int_{R_{1}}^{R_{2}} F_{H}(R) \mathrm{d} r .
$$

This scalable design of the shoe also allows the direction of the wheels to be changed so the shoe can similarly provide a forward motion. This allows us to put one shoe on each foot where each foot generates an opposite horizontal motion.
Using two shoes would provide the rehabilitative split-motion effects in an environment that most closely resembles walking over ground and would allow the greatest ground motion differential.

2) Gear Train and Magnetic Particle Brake: Our new design alleviates the largest deficiency of the previous shoe: the large motion variability generated and the jerkiness during each step. To overcome this limitation, the angular velocity of the wheels in our new design can be controlled by a braking system. This braking system consists of a gear train and a 0-24V small magnetic particle brake, which both needs to be strong enough to resist any motion as a person is stepping on the shoe. The magnetic particle brake is essentially a voltage actuated clutch; the more voltage is applied, the harder two internal plates push together to impede shaft rotation. The strength of the gear train and brake is demonstrated in the included video where, at first, it is enabled at full strength preventing any motion and then disabled allowing the wheels to rotate.

As shown in Figure 4, the front axle is mated to the rear axle with a chain and sprockets. As the wearer applies a vertical downward force onto any or all of the four wheels, the combined torque is fed from the rear axle through the gear train and to the brake. The maximum torque exerted by the wheels onto the rear axle can be estimated using Equation 1 combined with Equation 4,

$$
T=F_{H, a v g} R_{2} \text {. }
$$

Two sets of Miter gears were used to redirect the gear train for a space efficient fit. Three spur gears with 4:1 gear reductions each were used to reduce the torque applied by the GEMS wheels by a factor of 64 . A $1 \mathrm{lb}$-in magnetic particle brake was selected to apply resistance to the reduced torque. During the brake selection, the friction resistance forces and resistance forces of the spring actuated wheel reset mechanism were taken into account. Note that we chose to only use a 

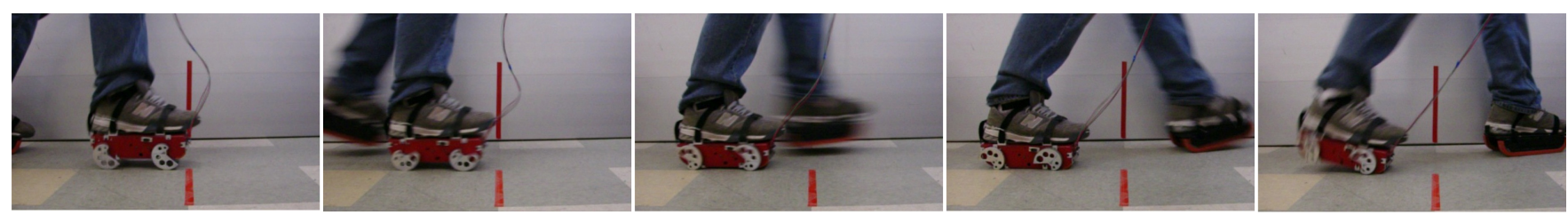

Fig. 6. GEMS from heel contact until toe off. The GEMS was measured to have a smooth horizontal backward motion of $6^{\prime \prime}(15.2 \mathrm{~cm})$. Note that one floor tile is $12^{\prime \prime}(30.5 \mathrm{~cm})$. These images are taken from the included video.

brake since the horizontal force is generated by the redirected downward force, a spring can generate the return force, and a motor capable of generating the necessary torque would require too much power and weight.

3) Wheel Reset Mechanism: In order for the shoe dynamics to be identical every step, the GEMS resets the wheel position using the spring mechanism shown in Figure 4. This reset mechanism consists of two extension springs, nylon strings, and a pulley which is attached to a gear axle. As the wearer applies a vertical downward force on the wheels during the stance phase, the rotation of the wheels causes the pulley to rotate, pulling the two extension springs apart. The potential energy stored in the extension springs is released during the swing phase so the wheels are rotated back to their initial position and ready for a subsequent step. The springs were selected so their force can overcome all internal friction of the system while having a sufficient stretch length and stiffness.

4) Electronics: The GEMS varies the motion resistance through the magnetic particle brake using an op-amp circuit in conjunction with a BS2p24 microprocessor (Figure 4). Depending on what point in the gait cycle the wearer is, variable resistance is applied to the GEMS. Instances in the gait cycle are identified by using a rotational potentiometer and an accelerometer. While the potentiometer recognizes the wheel rotation, the accelerometer recognizes when heel contact and toe off occur. Heel contact is measured by the jerk applied to the wheel and frame of the GEMS, and toe off is determined when the shoe has tipped forward by 30 degrees. In order to easily reprogram the on-board microprocessor, an external RS232 connection was mounted outside the side of the GEMS. All electronics are powered by a small battery pack, which the user wears on their hip.

5) GEMS Frame: The GEMS symmetric frame shape was chosen so it could be used in both directions as the wheels can be repositioned. It is made out of light and strong $3 / 16^{\prime \prime}(0.5 \mathrm{~cm})$ fiberglass and held together with various aluminum brackets. Two rubber pieces were added to the lower front and back corner of the shoe so the wearer could effectively create a solid heel contact and toe off.

6) Shoe Straps: During usage, the wearer's shoe is strapped down to the top of the GEMS. These straps were designed after a traditional sandal design, rigidly supporting the whole foot with minimal straps. Velcro straps were utilized for quick strapping and unstrapping of the wearer's shoe to the GEMS.

7) Opposite Leg Support Platform: Because the GEMS is $2^{\prime \prime}(5 \mathrm{~cm})$ off the ground, a supporting platform of equal height and weight for the opposite foot was constructed. The support platform was designed to be the exact same dimensions, weight, and fastening style to eliminate any unnecessary asymmetries. This platform was made with a thick rubber sole to maximize friction and stepping smoothness.

\section{RESULTS AND DisCUSSION}

This functional motion controlled Gait Enhancing Mobile Shoe (GEMS) design resulted in a total shoe dimension of $10.5^{\prime \prime} \times 4.5 \times 2$ 2 and a total weight of $4.5 \mathrm{lb}(2 \mathrm{~kg})$. Most of the total GEMS height and weight comes from the gear train and the magnetic particle brake responsible for controlling the wheel rotation. Considering that the average walking sneaker weighs about $1.5-2 \mathrm{lb}$, this final weight is on the heavy side of the acceptable range for a shoe of this purpose, but a lighter version is planned for long-term testing. Optimizing the wheel shape, spring forces, and reset mechanism would help to reduce the overall weight and most likely reduce the overall height of the shoe.

The motion controlled GEMS successfully applied a horizontal backward motion to the user's foot during the stance phase. The total backward motion distance averaged $6^{\prime \prime}(15.2 \mathrm{~cm})$ per step. This motion, which is shown in Figure 6 and in the included video, was observed to be continuous and smooth. The horizontal backward motion was comparable to the natural smoothness of a split-belt treadmill.

While the previous GEMS design [12] successfully slid the wearer's foot backward $10^{\prime \prime}(25 \mathrm{~cm})$, much of the backward motion was similar to a sliding motion. Using the Archimedean spiral wheel shape design, the new GEMS continues to utilize the wearer's vertical downward force due to the wearer's weight in the stance phase and converts it into a horizontal backward motion that can be controlled.

The sudden and large displacement in the previous GEMS also has a tendency to activate the wearer's natural instincts to restore balance making the walking pattern somewhat unnatural. This again has been corrected in the new GEMS design, allowing a shorter and smoother backward foot displacement as shown in Figure 7. The backward displacement of the foot in the new GEMS follows a constant and smooth $7.92 \mathrm{in} / \mathrm{sec}$ $(20.12 \mathrm{~cm} / \mathrm{sec})$ velocity.

Another interesting observation is that the large displacement distance from the previous GEMS resulted in a slowed down total walking speed, which can be corrected using this GEMS version. Since the motion is only a $6^{\prime \prime}(15.2 \mathrm{~cm})$ with a smooth displacement, a second shoe pushing in the opposite 


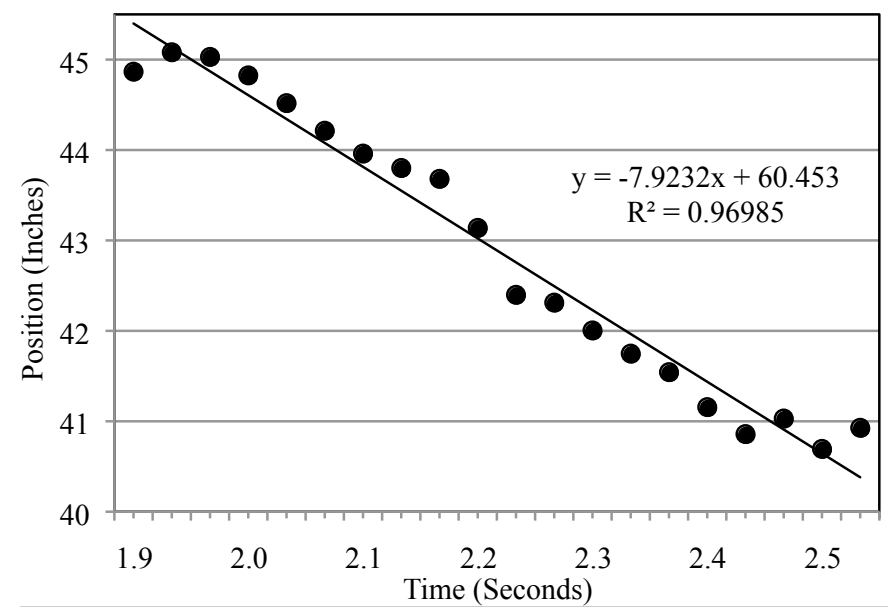

Fig. 7. Position of the shoe during stance phase. The motion is significantly more constant and smoother than the previous version, which should enable individuals to adapt to the motion. Note that the Archimedean wheels were not aligned to provide the maximum backward motion, thus only $4^{\prime \prime}(10.2 \mathrm{~cm})$ of motion was captured for this data.

direction will enable the desired difference between the feet. Since one foot moves forward and the other backward, the net progression of the individual will be the same as walking without any GEM shoes.

\section{CONCLUSIONS AND Future Work}

We successfully designed and constructed a functional gait enhancing mobile shoe (GEMS). As demonstrated in the submitted video it is capable of producing smooth and continuous backward motion during the stance phase of the gait cycle once loaded vertically upon heel contact. Although the previous version of the GEMS effectively showed some after-effects in the wearer's gait comparable to previous splitbelt rehabilitation studies, it was unnaturally jerky pushing the wearer's foot back in a sudden motion analogous to slipping on ice. This type of sudden motion enables a person's recovery and balancing instincts, thus producing an unnatural feel. This unnatural motion was greatly reduced in this version of the motion controlled GEMS model.

Our improved model is easily adjustable to different horizontal push length, force, speed and direction by simply adjusting the wheel size, wheel shape, and magnetic particle brake resistance. This adjustability in behavior of the GEMS makes testing for various situations possible.

Even though many drawbacks of the previous design were corrected there are numerous improvement opportunities in the design. Such improvements include a better choice of materials, adding a middle point of contact to ease the transition between wheels, and optimization. The optimization could balance the forces between the gear train, magnetic particle brake, wheel shape, frictional forces, and reset mechanism. The Archimedean spiral wheel shape alone is an interesting and agile aspect of the GEMS and is open to a detailed analysis, possibly resulting in shaping the wheel in such a way where desired horizontal forces are produced at specific instances of rotation.
Although still too heavy and unreliable for actual testing on persons with asymmetric gait, the GEMS as developed is sufficient enough for healthy subject test trials revealing if in fact this design can affect more positive gait altering effects than the previous version and comparable to previous split-belt research.

\section{ACKNOWLEDGMENT}

The authors thank the USF machine shop and Ron Rizzo from Western Kentucky University. The project described was supported by Award Number R21HD066200 from the Eunice Kennedy Shriver National Institute of Child Health and Human Development, but does not necessarily represent the official views of the National Institutes of Health.

\section{REFERENCES}

[1] D. Reisman, R. Wityk, and A. Bastian, "Split-belt treadmill walking adaptation in post-stroke hemiparesis," J. Neurologic Physical Therapy, vol. 29, p. 196, 2005.

[2] T. Lam, M. Anderschitz, and V. Dietz, "Contribution of feedback and feedforward strategies to locomotor adaptations," J. Neurophysiol, vol. 95, pp. 766-773, 2006.

[3] S. Morton and A. Bastian, "Cerebellar Contributions to Locomotor Adaptations during Splitbelt Treadmill Walking," J. Neurosci., vol. 26 , no. 36, pp. 9107-9116, 2006.

[4] T. Lam, C. Wolstenholme, and J. F. Yang, "How do infants adapt to loading of the limb during the swing phase of stepping?" Journal of Neurophysiology, vol. 89, no. 4, pp. 1920-1928, 2003.

[5] D. Reisman, R. Wityk, K. Silver, and A. Bastian, "Locomotor adaptation on a split-belt treadmill can improve walking symmetry post-stroke," Brain, vol. 130, no. 7, pp. 1861-1872, 2007.

[6] J. Krakauer, M. Ghilardi, and G. Ghez, "Independent learning of internal models for kinematic and dynamic control of reaching." Nature Neuroscience, vol. 2, pp. 1026-1031, 1999.

[7] J.-S. Lou and J. R. Bloedel, "A new conditioning paradigm: Conditioned limb movements in locomoting decerebrate ferrets," Neuroscience Letters, vol. 84, no. 2, pp. 185 - 190, 1988.

[8] D. S. Reisman, R. Wityk, K. Silver, and A. J. Bastian, "Splitbelt treadmill adaptation transfers to overground walking in persons poststroke." Neurorehabil Neural Repair, vol. 23, no. 7, pp. 735-744, Sep 2009.

[9] R. Keamey, "Context dependence of intrinsic and reflex contributions to ankle stiffness," in Proc. of 1st International IEEE EMBS Conf. on Neural Engineering, March 2003, pp. 434-437.

[10] R. F. Reynolds and A. M. Bronstein, "The broken escalator phenomenon. aftereffect of walking onto a moving platform." Exp Brain Res, vol. 151, no. 3, pp. 301-308, Aug 2003.

[11] A. M. Bronstein, K. L. Bunday, and R. Reynolds, "What the "broken escalator" phenomenon teaches us about balance." Ann N Y Acad Sci, vol. 1164, pp. 82-88, May 2009.

[12] A. de Groot, R. Decker, and K. B. Reed, "Gait enhancing mobile shoe (GEMS) for rehabilitation," in Proc. Joint Eurohaptics Conf. and Symp. on Haptic Interfaces for Virtual Environment and Teleoperator Systems, March 2009, pp. 190-195.

[13] R. Grasso, L. Bianchi, and F. Lacquaniti, "Motor Patterns for Human Gait: Backward Versus Forward Locomotion," J. Neurophysiol, vol. 80, no. 4, pp. 1868-1885, 1998.

[14] J. Perry, Gait Analysis: Normal and pathological function, N. S. Inc., Ed. Thorofare, 1992, vol. 50

[15] C. Honeycutt, J. Sushko, and K. B. Reed, "Asymmetric passive dynamic walker," in Proc. IEEE Int. Conf. Rehabilitation Robotics, 2011, in press.

[16] D. Reinkensmeyer, J. Emken, and S. Cramer, "Robotics, motor learning, and neurologic recovery," Annu. Rev. Biomed. Eng., vol. 6, pp. 497-525, 2004.

[17] M. Whittle, Gait Analysis: An Introduction. Elsevier Health Sciences, 1998.

[18] K. L. Bunday and A. M. Bronstein., "Visuo-vestibular influences involved in the broken escalator gait aftereffect," Gait and Posture, vol. 21, p. S23, 2005. 